\title{
Multi-Catalytic Approach to One-Pot Stereoselective Synthesis of Sec- ondary Benzylic Alcohols
}

\author{
Alessandra Casnati, $\ddagger$ Dawid Lichosyt, $\$$ Bruno Lainer, Lukas Veth, and Paweł Dydio* \\ University of Strasbourg, CNRS, ISIS UMR 7006, 8 allée Gaspard Monge, 67000 Strasbourg, France \\ Keywords: multi-catalysis, sequential catalysis, stereoselective synthesis, transition metal-catalysis, efficiency.
}

\begin{abstract}
One-pot multi-step procedures bear the potential to rapidly build up molecular complexity while avoiding the wasteful and costly isolations and purifications of consecutive intermediates. Here we report multi-catalytic protocols that convert alkenes, unsaturated aliphatic alcohols, and aryl boronic acids into secondary benzylic alcohols with high stereoselectivities under sequential catalysis that integrates alkene cross-metathesis, isomerization, and nucleophilic addition. Because each transformation of the sequence is executed by an independent catalyst, without any catalytic cross-reactivity, allylic alcohols bearing a prochiral double bond can be converted to any stereoisomer of the product with high stereoselectivity $(>98: 2 \mathrm{er}$ and $>20: 1 \mathrm{dr}$ ). Overall, with the aid of up to four catalysts operating in a single vessel, the protocols directly convert simple starting materials into a range of value-added products with high stereocontrol and excellent material efficiency, demonstrating both the efficacy and the advantages of the one-pot synthesis employing multiple transition-metal catalysts.
\end{abstract}

Despite substantial progress, the preparation of finechemicals and pharmaceuticals remains resource-intensive, raising serious sustainability and environmental concerns. ${ }^{1,2}$ According to the analysis of organic processes performed by GlaxoSmithKline, ${ }^{3}$ organic solvents account for $80 \%$ of all material consumption, except of water, and $75 \%$ of the energy use as well as $50 \%$ of the greenhouse gas emissions. Because the bulk of organic solvents is used for isolation and purification of synthetic intermediates, rather than as the medium of the actual chemical reactions, procedures that execute multiple synthetic steps in a one-pot fashion are advantageous from the efficiency standpoint.4-20 The economy of one-pot synthesis ${ }^{21}$ that connects stoichiometric and catalytic reactions, with or without limited intermediary work-up, proved highly beneficial for the synthesis of complex molecules, ${ }^{22}$ including natural products and pharmaceuticals. ${ }^{23-25}$ However, the development of one-pot protocols with multiple transition-metal catalyzed reactions operating in one vessel remains a challenge. ${ }^{26-33}$ The prospective cross-reactivity of the catalysts, including the exchange of ligands and formation of a mixture of complexes, is likely to hinder the required activity. ${ }^{15}$ The issues are further enhanced in asymmetric catalysis, where any cross-reactivity is likely to deteriorate the stereoselectivity of the intended transformations. ${ }^{34-39}$

Stereoselective synthesis has been facilitated by the development of transition metal-catalyzed asymmetric hydrogenation..$^{40-42}$ For instance, secondary benzylic alcohols, a prevalent moiety in biologically active molecules and valuable building blocks in synthesis, ${ }^{43-47}$ are readily prepared by enantioselective hydrogenation of aryl ketones in the presence of chiral Ru-complexes developed by Noyori. ${ }^{40}$ However, the preparation of ketones often requires a series of wasteful stochiometric oxidation and addition reactions, impeding the process efficiency (Figure 1a).

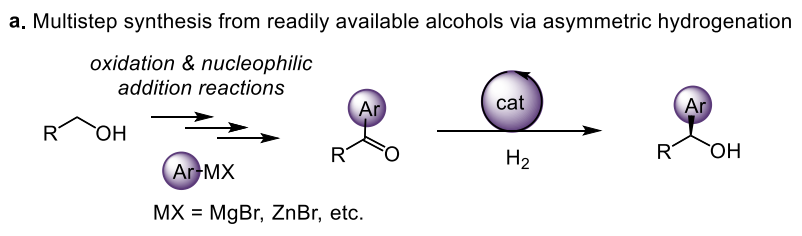

b. Two-step synthesis from readily available alcohols via asymmetric addition

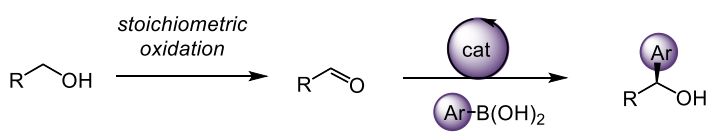

c. One-pot synthesis from available unsaturated alcohols \& alkenes (this work) - unsaturated alcohols:<smiles>[R]C=C[14CH2]CO</smiles><smiles></smiles>

- alkenes:
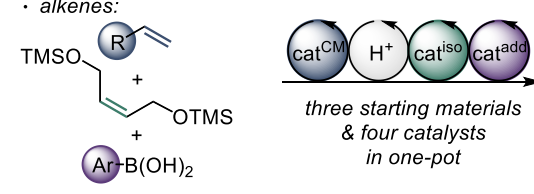
$\&$ four catalysts in one-pot
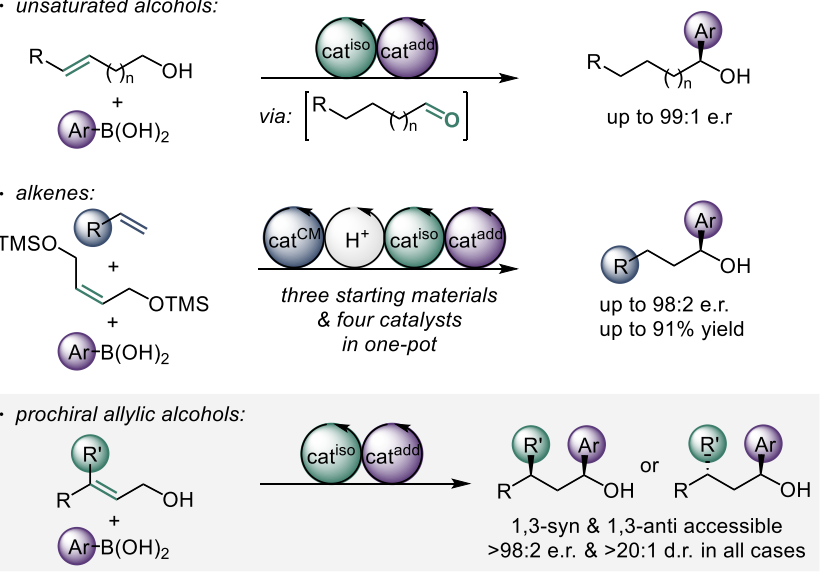

Figure 1. Context and the current study: stereoselective synthesis of secondary benzylic alcohols from readily available starting materials.

Asymmetric transformations that form a new stereogenic center and build up the molecular scaffold by connecting two building blocks are attractive. For instance, the 
enantioselective addition of an aryl nucleophile to an aldehyde to form a secondary benzylic alcohol ${ }^{48}$ represents an appealing alternative to the stepwise synthesis involving the enantioselective hydrogenation (Figure 1b). However, in this case, the aldehyde starting material typically also needs to be prepared from an accessible alcohol or carboxylic acid, burdening the process efficiency.

Within the framework of our program to target the inefficiency issues of organic synthesis with the aid of multi-catalytic systems, ${ }^{49}$ we propose a strategy to access enantioenriched secondary benzylic alcohols directly from aliphatic alcohols bearing an unsaturated bond. Such starting materials are both common motifs in bio-derived materials (e.g., terpenols) and readily accessible from abundant alkenes and simple alkenols by olefin cross-metathesis. ${ }^{50,51}$ In this context, we also consider alkenes and unsaturated alcohols as attractive redox-neutral surrogates of aliphatic aldehydes, ${ }^{52-55}$ given the alkene cross-metathesis, double bond isomerization, ${ }^{56}$ and the subsequent enantioselective addition reaction ${ }^{48}$ might be executed in situ with the aid of compatible catalysts.

Here we report broadly applicable multi-catalytic transition-metal protocols that execute redox-neutral transformations for a series of alkenes, unsaturated alcohols, and aryl boronic acids to furnish varied secondary benzylic alcohols in high stereoselectivity, with up to 99:1 er, dr >20:1, and $91 \%$ yield (Figure 1c). The protocols exploit a relay of up to 4 catalysts - 3 transition metal-complexes and a Brønsted acid - operating sequentially in the same reaction mixture. We showed that not only the one-pot protocol is operationally simpler and $\sim 3$-fold less resource-intensive than the stepwise synthesis, but also that the overall yield of the product is increased ( $77 \%$ versus $43 \%$ ) thanks to preventing cumulative losses of the materials during subsequent isolations and purifications of the intermediates.

We initiated the study by validating the possibility to conduct in situ both the isomerization of unsaturated alcohols and the addition of aryl boronic acids to aldehyde intermediates. In exploratory experiments, we found that model substrates, trans-2-hexenol 1a and phenylboronic acid 2a, reacted to furnish racemic secondary benzylic alcohol 3aa, 1-phenyl-1-hexanol in 84\% yield in the presence of $[\operatorname{Ir}(\operatorname{cod}) \mathrm{Cl}]_{2}\left(\right.$ Iriso1) and $\left[\mathrm{Rh}(\mathrm{cod})\left(\mathrm{CH}_{3} \mathrm{CN}\right)_{2}\right]\left(\mathrm{BF}_{4}\right)_{2}\left(\mathbf{R h}^{\text {iso1 }}\right)$ (Figure 2a).48,56 The control experiments confirmed the active role of both complexes. The reactivity was completely suppressed or substantially diminished when either the rhodium or the iridium complex was not present $(<2 \%$ or $31 \%$ yield, respectively).

Subsequent experimentation toward an enantioselective variant of the transformation indicated the requirement to exchange the Rh-catalyst for the Ru-complex bearing chiral Me-BIPAM, ( $\mathbf{R} \mathbf{u}^{\text {add }}$, Figure $\left.2 \mathrm{~b}\right)$; the latter being a privileged ligand developed by Yamamoto and Miyaura for enantioselective Rh- or Ru-catalyzed reactions, including 1,2-addi tion reactions. $18,57-62$ We found that although a Rh-catalyst bearing chiral diene ligand $\mathbf{R} \mathbf{h}^{\text {add2}} / \mathbf{c o d}^{*}$, broadly investigated by Hayashi, Carreira, and others, ${ }^{48,63-65}$ proved active in the model reaction of $\mathbf{1 a}$ and $\mathbf{2 a}$, product $3 \mathbf{a a}$ was formed in modest enantioselectivity, with up to 70:30 er, independently of the reaction conditions. The evaluation of other Rh-complexes bearing N-sulfinyl chiral sulfur-

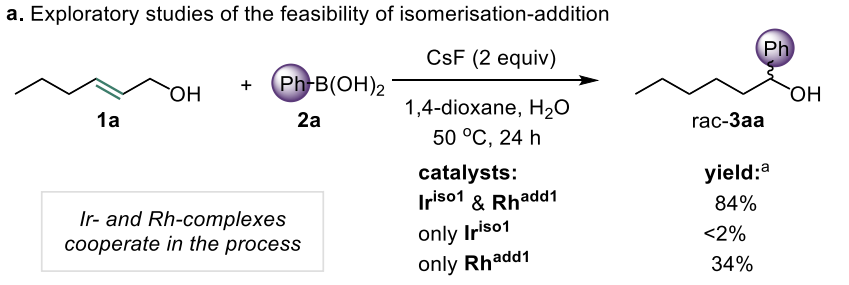

b. Study of enantioselective reaction with different isomerisation catalysts

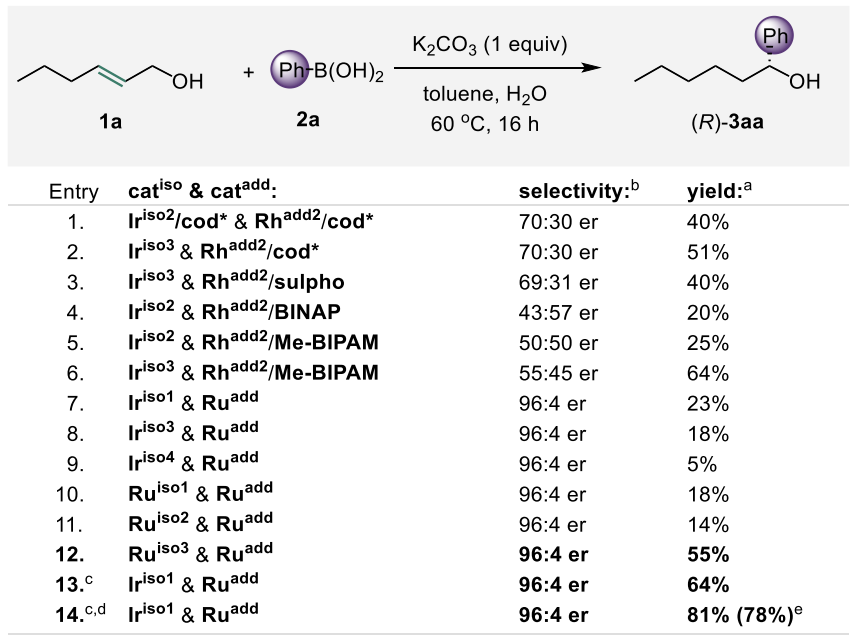

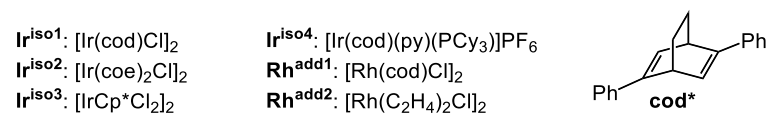

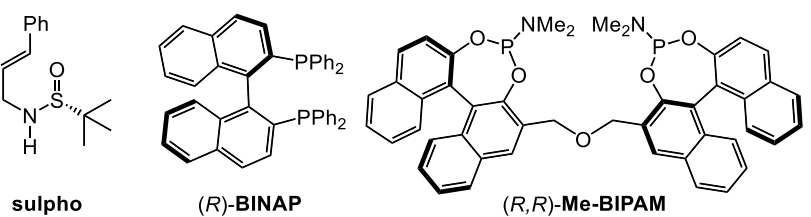

$(R)$-BINAP

$\mathbf{R u}^{\text {add. }}\left[\mathrm{Ru} \text { (cymene) } \mathrm{Cl}_{2}\right]_{2} /$ Me-BIPAM
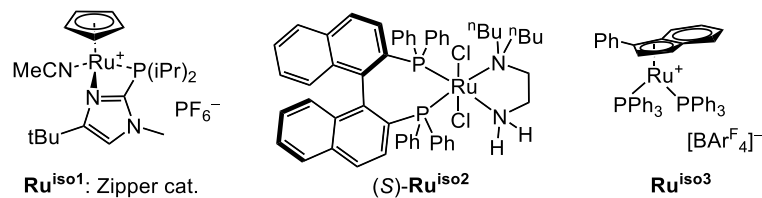

$\mathbf{R u}^{\text {iso1: }}$ Zipper cat

$(S)-\mathbf{R u}^{\text {iso2 }}$

$\mathrm{Ru}^{\mathrm{iso3}}$

Figure 2. Studies toward dual-catalytic enantioselective conversion of linear allylic alcohols to secondary benzylic alcohols. a Yields determined by ${ }^{1} \mathrm{H}$ NMR analysis with 1,3,5trimethoxybenzene as an internal standard. $\mathrm{b}$ Enantiomeric ratios determined by GC or SFC analysis on a chiral stationary phase. For further details, see the SI. c A mixture of 1a, $\left[\operatorname{Ir}(\operatorname{cod}) \mathrm{Cl}_{2}\right.$, and $\mathrm{K}_{2} \mathrm{CO}_{3}$ in toluene $(1 \mathrm{~mL})$ and water $(0.15 \mathrm{~mL})$ was first kept at $30^{\circ} \mathrm{C}$ for $2 \mathrm{~h}$, followed by the addition of $2 \mathrm{a}(3$ equiv), and $(R, R)$-Ruadd ( $6.0 \mathrm{~mol} \%)$ in toluene ( $1 \mathrm{~mL}$ ), and kept at $60{ }^{\circ} \mathrm{C}$ for $6 \mathrm{~h}$. d $0.125 \mathrm{~mol} \%$ Ir. e Yield of isolated material.

olefin $^{66,67} \mathbf{R h}^{\text {add2 }}$ /sulpho or phosphine ${ }^{63} \mathbf{R h}^{\text {add2 }}$ /BINAP ligands, known for their activity in Rh-catalyzed 1,4-addition, did not secure any highly enantioselective protocol. In sharp contrast, the reaction of $\mathbf{1 a}$ and $\mathbf{2 a}$ in the presence of Iriso1 and $(R, R)$-Ru ${ }^{\text {add }}, 58$ furnished product $(R)$-3aa in high er of $96: 4$, albeit in a low yield of $23 \%$. The use of other isomerization catalysts ${ }^{56,68-74}$ in place of Iriso1 enabled the formation of $(R)$-3aa in higher yields. The reaction in the presence of $\mathbf{R} \mathbf{u}^{\text {iso } 3}$ and $(R, R)$-Ruadd furnished the product in $55 \%$ yield and 96:4 er, executing the envisioned system of 
merging isomerization and enantioselective 1,2-addition reactions. Further experiments revealed that the presence of $\mathbf{R} \mathbf{u}^{\text {add }}$ complex partially inhibits the isomerization activity of Iriso1. Fortunately, when 1a was shortly incubated in the presence of just $0.125 \mathrm{~mol} \%$ Iriso1, prior to the addition of $\mathbf{2 a}$ and $(R, R)-\mathbf{R} \mathbf{u}^{\text {add }}$, the target product $(R)$-3aa was formed in 81\% yield and 96:4 er.

The identified protocol proved applicable to a substantial range of aryl boronic acids and alkenylic alcohols (Figure 3). The reactions of 1a with phenylboronic acid derivatives containing an electron-donating or an electron-withdrawing group in the para- or meta-position of the phenyl ring $(\mathbf{2 b}-\mathbf{2 i}, \mathbf{2 k - 2 n})$, as well as those with a sizeable aryl moiety (2o-2r) formed the product with 92:8 to 99:1 er and up to 93\% yield. The reactions for aryl boronic acids bearing either a strongly electron-withdrawing group or steric hin-

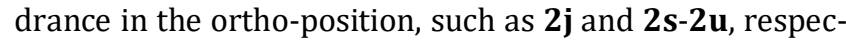
tively, furnished the products in modest yields (up to 35\%) and moderate enantioselectivity ( $<79: 21 \mathrm{er}$ ), indicating the limitations of the protocol. Noteworthy are the transformations involving heteroaryl derivatives, including dibenzothiophene 2o and 1,3-benzodioxole 2p, which delivered secondary benzylic alcohols in 95:5 er and $44-66 \%$ yields. Alcohols containing a more remote double bond, such as homoallylic 3-hexenol $\mathbf{1 b}$ and 5-hexenol 1c, are also suitable substrates for this sequential transformation; however, when the Zipper catalyst ( $\mathbf{R} \mathbf{u}^{\text {iso2 }}$, cf. Figure $2 \mathrm{~b}$ ) was used in place of Iriso1 the products were formed in higher yields (93\% and $90 \%$, respectively), maintaining high enantioselectivity (96:4 er). It is worth noting that the presence of $\mathbf{R} \mathbf{u}^{\text {iso2 }}$ bearing an achiral phosphine ligand does not erode the enantioselectivity of the step executed by chiral $\mathbf{R} \mathbf{u}^{\text {add }}$ catalyst, demonstrating the key compatibility between the catalysts.

Because cross-metathesis represents a convenient approach to install an alkenylic alcohol moiety on olefins, ${ }^{50,51}$ we next sought a protocol that would enable the direct assembly of a secondary benzylic alcohol from an alkene, a simple alkenylic alcohol, and an aryl boronic acid in a onepot fashion. Such a method would be attractive due to the increase in the structural diversity of the products, by using combinations of readily accessible building blocks. However, the requirement of the compatibility of 3 transitionmetal catalysts in the series of 3 subsequent processes represents a major challenge. ${ }^{15}$ Cross-inhibition issues aside, any ligand exchange processes between a metathesis or an isomerization catalyst and a chiral catalyst that operates in the final 1,2-addition step are likely to deteriorate the overall stereoselectivity of the process. ${ }^{28}$

Initial experiments indicated that a cross-metathesis reaction of alkene 4a and cis-2-butene-1,4-diol (5) in the presence of the o-tolyl Hoveyda-Grubbs Catalyst ${ }^{\circledR}$ M721(Ru ${ }^{\mathrm{CM}}$, often referred to as the Stewart-Grubbs catalyst ${ }^{75,76}$ followed in situ by the isomerization-addition sequence with $\mathbf{2 a}$, $\mathbf{R} \mathbf{u}^{\text {iso1, }}$, and $(R, R)$-Ru $\mathbf{u}^{\text {add }}$ furnished the target benzylic alcohol $(R)$-6aa in 98:2 er (Figure 4). High stereoselectivity of the overall reaction confirmed the critical compatibility of chiral $\mathbf{R} \mathbf{u}^{\text {add }}$ with the other catalysts in the sequence. However, because of the limited conversion of alkene 4a to allylic alcohol intermediate $\mathbf{7}$, even in the presence of a large excess $\mathbf{5}$, product $(R)$-6aa was formed in only

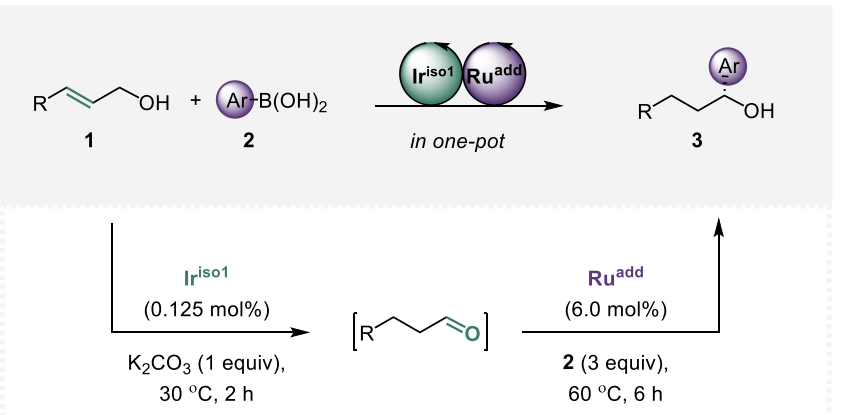

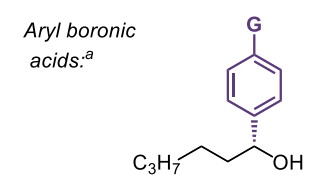

3aa: $\mathrm{G}=\mathrm{H}: 81 \%, 96: 4 \mathrm{er}$ 3ab: G $=$ OMe: $92 \%, 96: 4$ er 3ac: $\mathrm{G}=$ Me: $87 \%, 97: 3 \mathrm{er}$ 3ad: $\mathrm{G}=\mathrm{iPr}: 80 \%, 96: 4 \mathrm{er}$ 3ae: $\mathrm{G}=\mathrm{SMe}: 83 \%$, 95:5 er 3af: $\mathrm{G}=\mathrm{Ph}: 54 \%, 95: 5 \mathrm{er}$ $72 \%$, , $92: 8$ er 3ag: $\mathrm{G}=\mathrm{F}: 93 \%, 96: 4 \mathrm{er}$ 3ah: $\mathrm{G}=\mathrm{Cl}: 83 \%, 95: 5 \mathrm{er}$ 3ai: $\mathrm{G}=\mathrm{Br}: 83 \%, 95: 5 \mathrm{er}$ 3aj: $\mathrm{G}=\mathrm{CF}_{3}: 30 \%, 60: 40 \mathrm{er}$

$$
\begin{aligned}
& \text { 3ar: } \mathrm{Ar}=2-\text { Naphthyl: } 86 \%, 98: 2 \mathrm{er} \\
& \text { 3as: } \mathrm{Ar}=1-\mathrm{Naphthyl}: 35 \%,{ }^{,} \text {79:21 er } \\
& \text { 3at: } \mathrm{Ar}=9-\text { Phenantryl: } 15 \%,{ }^{\mathrm{b}} 72: 28 \mathrm{er} \\
& \text { 3au: } \mathrm{Ar}=2 \text {-Tolyl: }<5 \%
\end{aligned}
$$

Alcohols bearing a remote double bond: ${ }^{a}$

$\widehat{ح O H}+\mathrm{PhB}(\mathrm{OH})_{2}$

$1 \mathrm{~b}$

2a
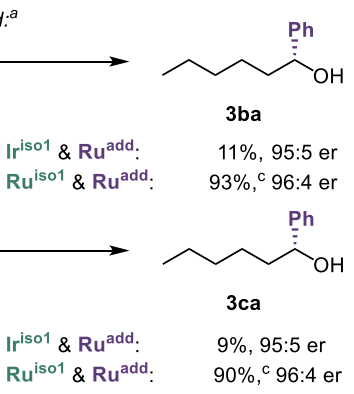

Figure 3. Substrate scope of dual-catalytic enantioselective conversion of linear alkenylic alcohols to secondary benzylic alcohols. a Reagents added to the reaction mixture subsequently without any work-up; yields determined by ${ }^{1} \mathrm{H}$ NMR analysis with a standard; yields of isolated material (column chromatography) are $\sim 3-9 \%$ lower; er determined by SFC analysis. b Upon addition $\mathbf{2}$ and Ruadd, the mixture was kept at $90^{\circ} \mathrm{C}$ for $24 \mathrm{~h}^{\mathrm{c}}{ }^{\mathrm{C}} \mathbf{R u}{ }^{\text {iso1 }}$ ( $2 \mathrm{~mol} \%$ ) was used in place of Iriso1.

up to $11 \%$ yield (Tables S1-S2 in the SI). Further investigation revealed that alkene $\mathbf{4 a}$ reacts more readily with $\mathbf{5}^{\prime}$, TMS-protected $\mathbf{5}$, in the presence of $\mathbf{R} \mathbf{u}^{\mathbf{C M}}$, forming $\mathbf{7}^{\prime}$ in a high yield ( $>95 \%$ by GC analysis). ${ }^{77}$ The latter is quickly and quantitatively deprotected to form alkenol $\mathbf{7}$ in the presence of catalytic trifluoroacetic acid. The acid is simply washed off with an aqueous base solution, and no workup is required for the subsequent isomerization-addition sequence. Such a one-pot protocol involving 4 catalysts, i.e., 3 different Ru-catalysts and a Brønsted acid, converts 4a, 5', and 2a to furnish the benzylic alcohol $(R)-6$ aa in $88 \%$ overall yield and 98:2 er. 


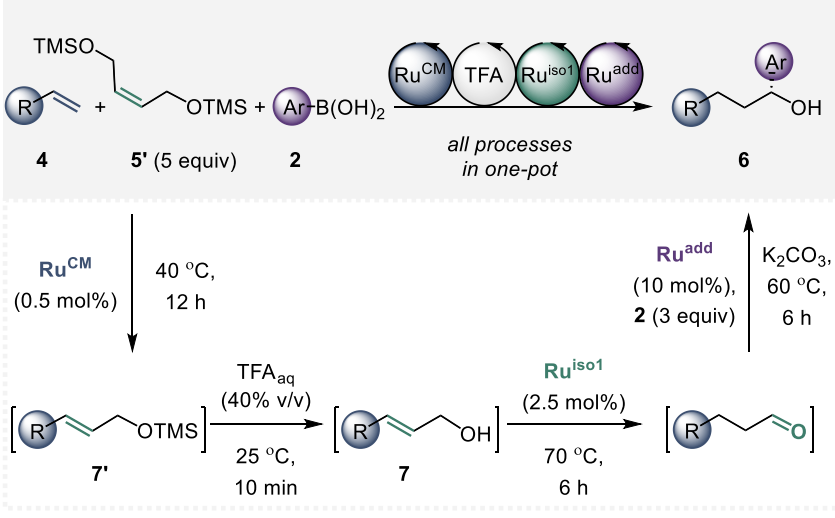

Substrate scope: ${ }^{a}$<smiles>CC(C)(C)CCCC(O)c1ccccc1</smiles>

$\overbrace{\mathrm{GH}}^{\mathrm{Ph}}$

6aa: $88 \%, 98: 2$ er

6ba: $\mathrm{G}=\mathrm{n}-\mathrm{C}_{7} \mathrm{H}_{15}: 65 \%, 97: 3 \mathrm{er}$ $(38 \%, 98: 2 \mathrm{er})^{\mathrm{b}}$ 6ca: $\mathrm{G}=\mathrm{Cy}: 72 \%, 97: 3 \mathrm{er}$

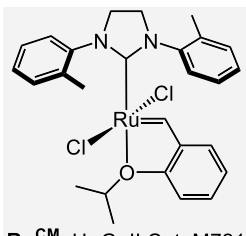<smiles>OC(CCCc1ccccc1)c1ccccc1</smiles><smiles>OC(CCc1ccc(Cl)cc1)c1ccccc1</smiles>

6ea: $\mathrm{G}=\mathrm{F}: 84 \%, 98: 2 \mathrm{er}$

6da: $53 \%, 97: 3$ er 6ea: $\mathrm{G}=\mathrm{F}: 84 \%, 98: 2 \mathrm{er}$
6fa: $\mathrm{G}=\mathrm{Cl}: 81 \%, 97: 3 \mathrm{er}$ 6ga: $\mathrm{G}=$ OAc: $90 \%, 97: 3 \mathrm{er}$<smiles>OC(CCc1cc(C(F)(F)F)cc(C(F)(F)F)c1)c1ccccc1</smiles>

6ha: $79 \%, 97: 3$ er

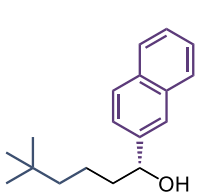

6ag: $90 \%, 97: 3 \mathrm{er}$

6ab: $\mathrm{G}=$ OMe: $84 \%, 98: 2$ er $\mathbf{6 a e}: \mathrm{G}=\mathrm{Me}: 91 \%, 98: 2 \mathrm{er}$ 6ac: $\mathrm{G}=\mathrm{SMe}: 87 \%, 96: 4$ er 6 af: $\mathrm{G}=\mathrm{Cl}: 80 \%, 95: 5 \mathrm{er}$ 6ad: $\mathrm{G}=\mathrm{F}: 85 \%, 98: 2 \mathrm{er}$

Figure 4. Multi-catalytic enantioselective sequential relay for conversion of alkenes to secondary benzylic alcohols. a Reagents added to the reaction mixture subsequently without any work-up (except for washing off TFA with water); yields determined by ${ }^{1} \mathrm{H}$ NMR analysis with a standard; yields of isolated material (column chromatography) are 3-9\% lower; enantioselectivity determined by SFC analysis; see the SI. ${ }^{b}$ Cis-2butene-1,4-diol 5 used in place of 5'; treatment with TFA was omitted.

The side-by-side experiments on a $2.4 \mathrm{mmol}$ scale of $\mathbf{4 a}$ for the synthesis of $(R)$-6aa proved that not only is the onepot protocol faster, more operationally simple, and $\sim 3$-fold less resource-intensive than the stepwise approach, but the final yield of the isolated material is also increased, i.e., $77 \%$ versus $43 \%$, respectively. Although the consecutive steps of the sequence occurred with similar GC yields in both cases, the one-pot protocol prevented cumulative losses of the material during subsequent isolations and purifications of the intermediates, illustrating an additional advantage of the approach (for details on the yields and amounts of resources used for each approach, see Tables S3-S4 in the SI).

The established protocol integrating alkene cross-metathesis, isomerization, and enantioselective addition is broadly applicable (Figure 4). A series of aliphatic alkenes, electron-rich or electron-deficient vinyl arenes, TMSprotected alkenol, and stereoelectronically varied aryl boronic acids reacted to form a range of secondary benzylic

alcohols in high enantioselectivities (er's > 95:5) and 5391\% overall yields.

Lastly, we focused on allylic alcohols bearing a prochiral double bond. The isomerization-addition sequence for 3substituted allylic alcohols constitutes an attractive strategy to produce the secondary benzylic alcohols bearing two stereocenters with a 1,3-relationship. We surmised that a method utilizing two different chiral catalysts that independently construct each stereogenic center would give access to all 1,3-syn and 1,3-anti stereoisomers of the product. However, the key requirement is the fully independent activity of both chiral catalysts.
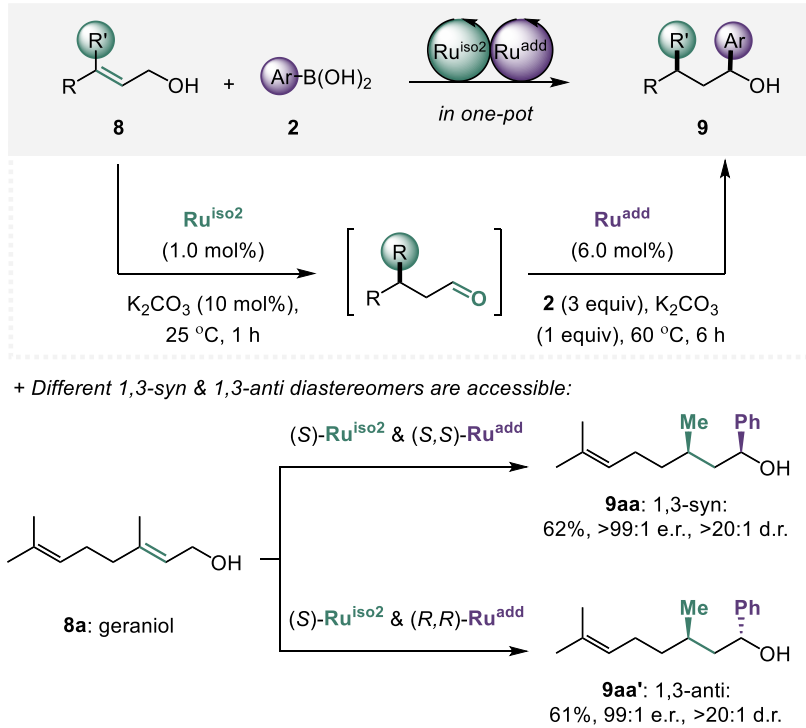

+ Configuration of double bond influences diastereoselectivity:

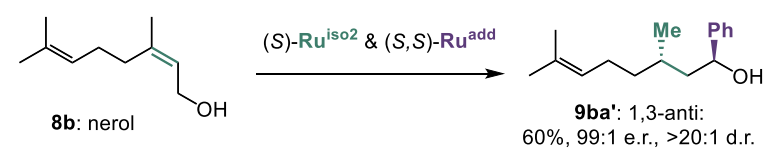

+ Different aryl boronic acids are compatible:
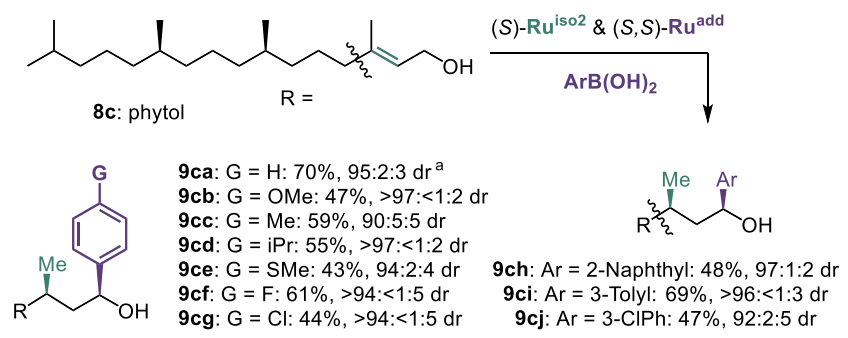

+ 3,4-Disubstituted allylic alcohols are compatible:

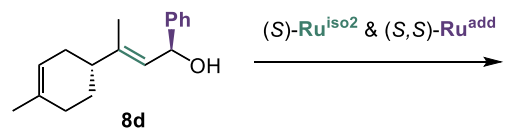<smiles>CC1=CCC(C(C)CC(O)c2ccccc2)CC1</smiles>

Figure 5. Dual-catalytic enantio- and diastereselective conversion of substitute allylic alcohols to secondary benzylic alcohols. Reagents added to the reaction mixture subsequently without any work-up (except for removal of EtOH under vacuum); stereoselectivity determined by SFC and GC analysis; yields correspond to the amounts of isolated major diastereomer (column chromatography); see the SI. a dr of 1,3-syn (major):1,3-syn(minor):sum of 1,3-anti products. 
The experimentation identified that pairing enantiomers of $\mathbf{R} \mathbf{u}^{\text {iso2 }}$ (cf. Figure 2), the isomerization catalyst reported by Ohkuma, ${ }^{72}$ and $\mathbf{R} \mathbf{u}^{\text {add }}$ enables the isomerization-addition sequence to form the products with high stereocontrol in a one-pot fashion (Figure 5). The catalysts proved to require different solvents to operate efficiently (i.e., ethanol and toluene, respectively; for details, see Figures S1-S4, Table S5). Therefore, the medium needs to be exchanged between the steps (evaporation under vacuum); albeit no resource-intensive work-up is needed. Importantly, the stereocontrol for the formation of each stereogenic center is solely determined by the catalyst involved. For instance, while the reaction of geraniol $\mathbf{8 a}$ and $\mathbf{2 a}$ in the presence of $(S)-\mathbf{R} \mathbf{u}^{\text {iso2 }}$ and $(S, S)$-Ruadd furnished 1,3-syn benzylic alcohol $(1 S, 3 R)-9$ aa with $>99: 1 \mathrm{er},>20: 1 \mathrm{dr}$, in 62\% yield, the same reaction but with $(R, R)$ - $\mathbf{R} \mathbf{u}^{\text {add }}$ in place of $(S, S)$ - $\mathbf{R} \mathbf{u}^{\text {add }}$ furnished 1,3-anti diastereomeric alcohol (1S,3S)-9aa', in similarly high 99:1 er, $>20: 1 \mathrm{dr}$, and 61\% yield. The diastereoselectivity of the transformation depends on the configuration of the double bond in the starting material. ${ }^{72}$ Nerol $\mathbf{8 b}$, the $(Z)$-analogue of geraniol 8a, reacted to form 1,3-anti $\mathbf{9 b a}$, i.e., the other diastereomer, in 99:1 er, >20:1 dr, and 60\% yield. Noteworthy, the isolated double bonds of starting materials 8a-8b remained intact in the corresponding products. Chiral phytol 8c, reacted with varied arylboronic acids to form the products in high stereoselectivity, i.e., $90-97 \%$ of the major stereoisomer, and from $44 \%$ to $70 \%$ yield. The presence of an additional chiral center next to the allylic alcohol moiety in the starting material seems not to disturb the reaction. $(+)$-Limonene derivative $\mathbf{8 d}$ reacted with $2 \mathbf{a}$ to form $9 \mathbf{d a}$ in $>20: 1 \mathrm{dr}$, and 41\% yield, expanding the scope of the system.

In conclusion, the herein disclosed methods enable the rapid modular stereoselective syntheses of a broad range of secondary benzylic alcohols from simple available starting materials. The strategy relies on the construction of the sequences of multiple catalytic reactions occurring consecutively in a single vessel. A transformation is executed with the aid of up to four catalysts and requires a single isolation and purification of the product. Overall, the approach simplifies the synthesis of target motifs, increases material efficiency, and limits cost, time, and waste associated with the standard stepwise procedures. In a greater perspective, the study highlights the synthetic potential of the multi-catalytic approaches to access increasingly complex architectures from simple starting materials.

\section{ASSOCIATED CONTENT}

Supporting Information. Details on experimental procedures for the catalytic reactions, including optimization data, spectroscopic data for the starting materials and the products. This material is available free of charge via the Internet at http://pubs.acs.org

\section{AUTHOR INFORMATION}

\section{Corresponding Author \\ *E-mail: dydio@unistra.fr}

\section{Author Contributions}

The manuscript was written through contributions of all authors. All authors have given approval to the final version of the manuscript.
¥AC and DL contributed equally.

\section{ACKNOWLEDGMENT}

This project has received funding from the European Research Council (ERC) under the European Union's Horizon 2020 research and innovation program (grant agreement no 804106). Further funding was provided by the French National Research Agency (ANR IdEx, ANR LabEx „Chemistry of Complex Systems", and a PhD fellowship for BL through the Programme d' Investissement d' Avenir under contract 17-EURE-0016). We are thankful to K. Hurej for preliminary experiments, and D. Leboeuf for the comments on the manuscript.

\section{REFERENCES}

(1) Sheldon, R. A. Metrics of Green Chemistry and Sustainability: Past, Present, and Future. ACS Sustain. Chem. Eng. 2018, 6 (1), 32-48. https://doi.org/10.1021/acssuschemeng.7b03505.

(2) Sheldon, R. A. The E Factor: Fifteen Years On. Green Chem. 2007, 9 (12), 1273. https://doi.org/10.1039/b713736m.

(3) Curzons, A. D.; Mortimer, D. N.; Constable, D. J. C.; Cunningham, V. L. So You Think Your Process Is Green, How Do You Know? - Using Principles of Sustainability to Determine What Is Green - a Corporate Perspective. Green Chem. 2001, 3 (1), 1-6. https://doi.org/10.1039/b007871i.

(4) Galván, A.; Fañanás, F. J.; Rodríguez, F. Multicomponent and Multicatalytic Reactions - A Synthetic Strategy Inspired by Nature. Eur. J. Inorg. Chem. 2016, 2016 (9), 1306-1313. https://doi.org/10.1002/ejic.201501287.

(5) Ambrosini, L. M.; Lambert, T. H. Multicatalysis: Advancing Synthetic Efficiency and Inspiring Discovery. ChemCatChem 2010, 2 (11), 1373-1380.

https://doi.org/10.1002/cctc.200900323.

(6) Patil, N. T.; Shinde, V. S.; Gajula, B. A One-Pot Catalysis: The Strategic Classification with Some Recent Examples. Org Biomol Chem 2012, 10 (2), 211-224.

https://doi.org/10.1039/С10В06432K.

(7) Quintard, A. Frontispiece: Iron-Based Multi-Catalysis: Eco-Compatible Alternative for Complex Molecules Synthesis. Chem. - Eur. J. 2021, 27 (1).

https://doi.org/10.1002/chem.202180163.

(8) Lee, J. M.; Na, Y.; Han, H.; Chang, S. Cooperative MultiCatalyst Systems for One-Pot Organic Transformations. Chem. Soc. Rev. 2004, 33 (5), 302. https://doi.org/10.1039/b309033g.

(9) Shao, Z.; Zhang, H. Combining Transition Metal Catalysis and Organocatalysis: A Broad New Concept for Catalysis. Chem. Soc. Rev. 2009, 38 (9), 2745. https://doi.org/10.1039/b901258n.

(10) Rueping, M.; Koenigs, R. M.; Atodiresei, I. Unifying Metal and Brønsted Acid Catalysis-Concepts, Mechanisms, and Classifications. Chem. - Eur. J. 2010, 16 (31), 9350-9365.

https://doi.org/10.1002/chem.201001140.

(11) Lohr, T. L.; Marks, T. J. Orthogonal Tandem Catalysis. Nat. Chem. 2015, 7 (6), 477-482.

https://doi.org/10.1038/nchem.2262.

(12) Fogg, D. E.; dos Santos, E. N. Tandem Catalysis: A Taxonomy and Illustrative Review. Coord. Chem. Rev. 2004, 248 (2124), 2365-2379. https://doi.org/10.1016/j.ccr.2004.05.012.

(13) Chapman, C.; Frost, C. Tandem and Domino Catalytic Strategies for Enantioselective Synthesis. Synthesis 2007, 2007 (1), 1-21. https://doi.org/10.1055/s-2006-950379.

(14) Hopkinson, M. N.; Sahoo, B.; Li, J.-L.; Glorius, F. Dual Catalysis Sees the Light: Combining Photoredox with Organo-, 
Acid, and Transition-Metal Catalysis. Chem. - Eur. J. 2014, 20 (14), 3874-3886. https://doi.org/10.1002/chem.201304823.

(15) Sancheti, S. P.; Urvashi; Shah, M. P.; Patil, N. T. Ternary Catalysis: A Stepping Stone toward Multicatalysis. ACS Catal. 2020, 10 (5), 3462-3489.

https://doi.org/10.1021/acscatal.9b04000.

(16) Chen, Z.-S.; Huang, X.-Y.; Chen, L.-H.; Gao, J.-M.; Ji, K. $\mathrm{Rh}(\mathrm{II}) / \mathrm{Pd}(0)$ Dual Catalysis: Regiodivergent Transformations of Alkylic Oxonium Ylides. ACS Catal. 2017, 7 (11), 7902-7907. https://doi.org/10.1021/acscatal.7b02909.

(17) Dhiman, S.; Mishra, U. K.; Ramasastry, S. S. V. One-Pot Trimetallic Relay Catalysis: A Unified Approach for the Synthesis of $\beta$-Carbolines and Other [ $c$ ]-Fused Pyridines. Angew. Chem. Int. Ed. 2016, 55 (27), 7737-7741. https://doi.org/10.1002/anie.201600840.

(18) Yamamoto, K.; Qureshi, Z.; Tsoung, J.; Pisella, G.; Lautens, M. Combining Ru-Catalyzed C-H Functionalization with Pd-Catalyzed Asymmetric Allylic Alkylation: Synthesis of 3-Allyl-3Aryl Oxindole Derivatives from Aryl $\alpha$-Diazoamides. Org. Lett. 2016, 18 (19), 4954-4957. https://doi.org/10.1021/acs.orglett.6b02423.

(19) Yin, X.-P.; Zeng, X.-P.; Liu, Y.-L.; Liao, F.-M.; Yu, J.-S.; Zhou, F.; Zhou, J. Asymmetric Triple Relay Catalysis: Enantioselective Synthesis of Spirocyclic Indolines through a One-Pot Process Featuring an Asymmetric $6 \pi$ Electrocyclization. Angew. Chem. Int. Ed. 2014, 53 (50), 13740-13745.

https://doi.org/10.1002/anie.201407677.

(20) Simmons, B.; Walji, A. M.; MacMillan, D. W. C. CycleSpecific Organocascade Catalysis: Application to Olefin Hydroamination, Hydro-Oxidation, and Amino-Oxidation, and to Natural Product Synthesis. Angew. Chem. Int. Ed. 2009, 48 (24), 43494353. https://doi.org/10.1002/anie.200900220.

(21) Hayashi, Y. Pot Economy and One-Pot Synthesis. Chem. Sci. 2016, 7 (2), 866-880. https://doi.org/10.1039/C5SC02913A.

(22) Yadav, R. N.; Srivastava, A. K.; Banik, B. K. One-Pot Strategy: A Highly Economical Tool in Organic Synthesis and Medicinal Chemistry. In Green Approaches in Medicinal Chemistry for Sustainable Drug Design; Elsevier, 2020; pp 353-425. https://doi.org/10.1016/B978-0-12-817592-7.00010-1.

(23) Ishikawa, H.; Suzuki, T.; Hayashi, Y. High-Yielding Synthesis of the Anti-Influenza Neuramidase Inhibitor (-)-Oseltamivir by Three "One-Pot" Operations. Angew. Chem. Int. Ed. 2009, 48 (7), 1304-1307. https://doi.org/10.1002/anie.200804883.

(24) Cameron, M.; Hoerrner, R. S.; McNamara, J. M.; Figus, M.; Thomas, S. One-Pot Preparation of 7-Hydroxyquinoline. Org. Process Res. Dev. 2006, 10 (1), 149-152. https://doi.org/10.1021/op0501545.

(25) Ho, G.-M.; Zulueta, M. M. L.; Hung, S.-C. Stereoselective One-Pot Synthesis of Polypropionates. Nat. Commun. 2017, 8 (1). https://doi.org/10.1038/s41467-017-00787-y.

(26) Allen, A. E.; MacMillan, D. W. C. Synergistic Catalysis: A Powerful Synthetic Strategy for New Reaction Development. Chem. Sci. 2012, 3 (3), 633. https://doi.org/10.1039/c2sc00907b.

(27) Trost, B. M.; Luan, X. Contemporaneous Dual Catalysis by Coupling Highly Transient Nucleophilic and Electrophilic Intermediates Generated in Situ. J. Am. Chem. Soc. 2011, 133 (6), 1706-1709. https://doi.org/10.1021/ja110501v.

(28) Romiti, F.; del Pozo, J.; Paioti, P. H. S.; Gonsales, S. A.; Li, X.; Hartrampf, F. W. W.; Hoveyda, A. H. Different Strategies for Designing Dual-Catalytic Enantioselective Processes: From Fully Cooperative to Non-Cooperative Systems. J. Am. Chem. Soc. 2019, 141 (45), 17952-17961. https://doi.org/10.1021/jacs.9b05464.
(29) Panteleev, J.; Zhang, L.; Lautens, M. Domino RhodiumCatalyzed Alkyne Arylation/Palladium-Catalyzed N Arylation: A Mechanistic Investigation. Angew. Chem. Int. Ed. 2011, 50 (39), 9089-9092. https://doi.org/10.1002/anie.201103692.

(30) Inamdar, S. M.; Shinde, V. S.; Patil, N. T. Enantioselective Cooperative Catalysis. Org. Biomol. Chem. 2015, 13 (30), 8116-8162. https://doi.org/10.1039/C5OB00986C.

(31) Chae, J.; Yun, J.; Buchwald, S. L. One-Pot Sequential CuCatalyzed Reduction and Pd-Catalyzed Arylation of Silyl Enol Ethers. Org. Lett. 2004, 6 (26), 4809-4812. https://doi.org/10.1021/ol048313c.

(32) Miura, T.; Tanaka, T.; Matsumoto, K.; Murakami, M. One-Pot Synthesis of 2,5-Dihydropyrroles from Terminal Alkynes, Azides, and Propargylic Alcohols by Relay Actions of Copper, Rhodium, and Gold. Chem. - Eur. J. 2014, 20 (49), 16078-16082. https://doi.org/10.1002/chem.201405357.

(33) Wang, H.; Denton, J. R.; Davies, H. M. L. Sequential Rhodium-, Silver-, and Gold-Catalyzed Synthesis of Fused Dihydrofurans. Org. Lett. 2011, 13 (16), 4316-4319. https://doi.org/10.1021/ol2016548.

(34) Pellissier, H. Recent Developments in Enantioselective Multicatalyzed Tandem Reactions. Adv. Synth. Catal. 2020, 362 (12), 2289-2325. https://doi.org/10.1002/adsc.202000210.

(35) Zhou, J. Recent Advances in Multicatalyst Promoted Asymmetric Tandem Reactions. Chem. - Asian J. 2010, 5 (3), 422 434. https://doi.org/10.1002/asia.200900458.

(36) Krautwald, S.; Sarlah, D.; Schafroth, M. A.; Carreira, E. M. Enantio- and Diastereodivergent Dual Catalysis: -Allylation of Branched Aldehydes. Science 2013, 340 (6136), 1065-1068. https://doi.org/10.1126/science.1237068.

(37) Krautwald, S.; Schafroth, M. A.; Sarlah, D.; Carreira, E. M. Stereodivergent $\alpha$-Allylation of Linear Aldehydes with Dual Iridium and Amine Catalysis. J. Am. Chem. Soc. 2014, 136 (8), 3020-3023. https://doi.org/10.1021/ja5003247.

(38) Sandmeier, T.; Krautwald, S.; Zipfel, H. F.; Carreira, E. M. Stereodivergent Dual Catalytic $\alpha$-Allylation of Protected $\alpha$ Amino- and $\alpha$-Hydroxyacetaldehydes. Angew. Chem. Int. Ed. 2015, 54 (48), 14363-14367.

https://doi.org/10.1002/anie.201506933.

(39) Banerjee, A.; Sahu, S.; Maji, M. S. Benzannulation of 2Alkenylindoles Using Aldehydes by Sequential Triple-Relay Catalysis: A Route to Carbazoles and Carbazole Alkaloids. Adv. Synth. Catal. 2017, 359 (11), 1860-1866. https://doi.org/10.1002/adsc.201700092.

(40) Noyori, R.; Ohkuma, T. Asymmetric Catalysis by Architectural and Functional Molecular Engineering: Practical Chemoand Stereoselective Hydrogenation of Ketones. Angew. Chem. Int. Ed. 2001, 40 (1), 40-73. https://doi.org/10.1002/15213773(20010105)40:1<40::AID-ANIE40>3.0.CO;2-5.

(41) Tang, W.; Zhang, X. New Chiral Phosphorus Ligands for Enantioselective Hydrogenation. Chem. Rev. 2003, 103 (8), 30293070. https://doi.org/10.1021/cr020049i.

(42) Noyori, R. Facts Are the Enemy of Truth-Reflections on Serendipitous Discovery and Unforeseen Developments in Asymmetric Catalysis. Angew. Chem. Int. Ed. 2013, 52 (1), 79-92. https://doi.org/10.1002/anie.201205537.

(43) Frishman, W. H. $\beta$-Adrenergic Blockade in Cardiovascular Disease. J. Cardiovasc. Pharmacol. Ther. 2013, 18 (4), 310319. https://doi.org/10.1177/1074248413484986.

(44) Kakei, H.; Nemoto, T.; Ohshima, T.; Shibasaki, M. Efficient Synthesis of Chiral $\alpha$ - And $\beta$-Hydroxy Amides: Application to the Synthesis of (R)-Fluoxetine. Angew. Chem. Int. Ed. 2004, 43 (3), 317-320. https://doi.org/10.1002/anie.200352431. 
(45) Fujima, Y.; Ikunaka, M.; Inoue, T.; Matsumoto, J. Synthesis of ( $S$ )-3-( $N$-Methylamino)-1-(2-Thienyl)Propan-1-Ol: Revisiting Eli Lilly's Resolution-Racemization-Recycle Synthesis of Duloxetine for Its Robust Processes. Org. Process Res. Dev. 2006, 10 (5), 905-913. https://doi.org/10.1021/op060118l.

(46) Tasker, S. Z.; Standley, E. A.; Jamison, T. F. Recent Advances in Homogeneous Nickel Catalysis. Nature 2014, 509 (7500), 299-309. https://doi.org/10.1038/nature13274.

(47) Duncton, M. A. J. Minisci Reactions: Versatile $\mathrm{CH}-$ Functionalizations for Medicinal Chemists. MedChemComm 2011, 2 (12), 1135. https://doi.org/10.1039/c1md00134e.

(48) Hayashi, T.; Yamasaki, K. Rhodium-Catalyzed Asymmetric 1,4-Addition and Its Related Asymmetric Reactions. Chem. Rev. 2003, 103 (8), 2829-2844.

https://doi.org/10.1021/cr020022z.

(49) Lichosyt, D.; Zhang, Y.; Hurej, K.; Dydio, P. Dual-CataIytic Transition Metal Systems for Functionalization of Unreactive Sites of Molecules. Nat. Catal. 2019, 2 (2), 114-122. https://doi.org/10.1038/s41929-018-0207-1.

(50) Koh, M. J.; Khan, R. K. M.; Torker, S.; Yu, M.; Mikus, M. S.; Hoveyda, A. H. High-Value Alcohols and Higher-OxidationState Compounds by Catalytic Z-Selective Cross-Metathesis. Nature 2015, 517 (7533), 181-186. https://doi.org/10.1038/nature14061.

(51) Xu, C.; Liu, Z.; Torker, S.; Shen, X.; Xu, D.; Hoveyda, A. H. Synthesis of $Z$ - or $E$-Trisubstituted Allylic Alcohols and Ethers by Kinetically Controlled Cross-Metathesis with a Ru Catechothiolate Complex. J. Am. Chem. Soc. 2017, 139 (44), 15640-15643. https://doi.org/10.1021/jacs.7b10010.

(52) Li, C.; Kan, J.; Qiu, Z.; Li, J.; Lv, L.; Li, C. Synergistic Relay Reactions To Achieve Redox-Neutral A-Alkylations of Olefinic Alcohols with Ruthenium(II) Catalysis. Angew. Chem. Int. Ed. 2020, 59 (11), 4544-4549. https://doi.org/10.1002/anie.201915218.

(53) Li, C.-C.; Wang, H.; Sim, M. M.; Qiu, Z.; Chen, Z.-P.; Khaliullin, R. Z.; Li, C.-J. Empowering Alcohols as Carbonyl Surrogates for Grignard-Type Reactions. Nat. Commun. 2020, 11 (1). https://doi.org/10.1038/s41467-020-19857-9.

(54) Zbieg, J. R.; Yamaguchi, E.; McInturff, E. L.; Krische, M. J. Enantioselective C-H Crotylation of Primary Alcohols via Hydrohydroxyalkylation of Butadiene. Science 2012, 336 (6079), 324327. https://doi.org/10.1126/science.1219274.

(55) Kim, S. W.; Zhang, W.; Krische, M. J. Catalytic Enantioselective Carbonyl Allylation and Propargylation via Alcohol-Mediated Hydrogen Transfer: Merging the Chemistry of Grignard and Sabatier. Acc. Chem. Res. 2017, 50 (9), 2371-2380. https://doi.org/10.1021/acs.accounts.7b00308.

(56) Cahard, D.; Gaillard, S.; Renaud, J.-L. Asymmetric Isomerization of Allylic Alcohols. Tetrahedron Lett. 2015, 56 (45), 6159-6169. https://doi.org/10.1016/j.tetlet.2015.09.098.

(57) Yamamoto, Y.; Kurihara, K.; Miyaura, N. Me-Bipam for Enantioselective Ruthenium(II)-Catalyzed Arylation of Aldehydes with Arylboronic Acids. Angew. Chem. Int. Ed. 2009, 48 (24), 4414-4416. https://doi.org/10.1002/anie.200901395.

(58) Yamamoto, Y.; Shirai, T.; Watanabe, M.; Kurihara, K.; Miyaura, N. Ru/Me-BIPAM-Catalyzed Asymmetric Addition of Arylboronic Acids to Aliphatic Aldehydes and $\alpha$-Ketoesters. Molecules 2011, 16 (6), 5020-5034. https://doi.org/10.3390/molecules 16065020 .

(59) Yamamoto, Y.; Kurihara, K.; Sugishita, N.; Oshita, K.; Piao, D.; Miyaura, N. Chiral Bis-Phosphoramidites Based on Linked-BINOL for Rhodium-Catalyzed 1,4-Addition of Arylboronic Acids to $\alpha, \beta$-Unsaturated Carbonyl Compounds. Chem. Lett. 2005, 34 (9), 1224-1225. https://doi.org/10.1246/cl.2005.1224.
(60) Kurihara, K.; Yamamoto, Y.; Miyaura, N. An N-Linked Bidentate Phosphoramidite Ligand (N-Me-BIPAM) for RhodiumCatalyzed Asymmetric Addition of Arylboronic Acids to N-Sulfonylarylaldimines. Adv. Synth. Catal. 2009, 351 (1-2), 260-270. https://doi.org/10.1002/adsc.200800631.

(61) Kurihara, K.; Sugishita, N.; Oshita, K.; Piao, D.; Yamamoto, Y.; Miyaura, N. Enantioselective 1,4-Addition of Arylboronic Acids to $\alpha, \beta$-Unsaturated Carbonyl Compounds Catalyzed by Rhodium(I)-Chiral Phosphoramidite Complexes. J. Organomet. Chem. 2007, 692 (1-3), 428-435. https://doi.org/10.1016/j.jorganchem.2006.04.042.

(62) Kurihara, K.; Yamamoto, Y.; Miyaura, N. A Chiral Bidentate Phosphoramidite (Me-BIPAM) for Rh-Catalyzed Asymmetric Hydrogenation of $\alpha$-Dehydroamino Esters, Enamides, and Dimethyl Itaconate. Tetrahedron Lett. 2009, 50 (26), 3158-3160. https://doi.org/10.1016/j.tetlet.2008.12.086.

(63) Tian, P.; Dong, H.-Q.; Lin, G.-Q. Rhodium-Catalyzed Asymmetric Arylation. ACS Catal. 2012, 2 (1), 95-119. https://doi.org/10.1021/cs200562n.

(64) Defieber, C.; Grützmacher, H.; Carreira, E. M. Chiral Olefins as Steering Ligands in Asymmetric Catalysis. Angew. Chem. Int. Ed. 2008, 47 (24), 4482-4502. https://doi.org/10.1002/anie.200703612.

(65) Johnson, J. B.; Rovis, T. More than Bystanders: The Effect of Olefins on Transition-Metal-Catalyzed Cross-Coupling Reactions. Angew. Chem. Int. Ed. 2008, 47 (5), 840-871. https://doi.org/10.1002/anie.200700278.

(66) Zhu, T.-S.; Jin, S.-S.; Xu, M.-H. Rhodium-Catalyzed, Highly Enantioselective 1,2-Addition of Aryl Boronic Acids to $\alpha$ Ketoesters and $\alpha$-Diketones Using Simple, Chiral Sulfur-Olefin Ligands. Angew. Chem. Int. Ed. 2012, 51 (3), 780-783. https://doi.org/10.1002/anie.201106972.

(67) Khiar, N.; Salvador, Á.; Chelouan, A.; Alcudia, A.; Fernández, I. "Sulfolefin": Highly Modular Mixed S/Olefin Ligands for Enantioselective Rh-Catalyzed 1,4-Addition. Org. Biomol. Chem. 2012, 10 (12), 2366. https://doi.org/10.1039/c2ob07132k.

(68) Grotjahn, D. B.; Larsen, C. R.; Gustafson, J. L.; Nair, R.; Sharma, A. Extensive Isomerization of Alkenes Using a Bifunctional Catalyst: An Alkene Zipper. J. Am. Chem. Soc. 2007, 129 (31), 9592-9593. https://doi.org/10.1021/ja073457i.

(69) Larsen, C. R.; Erdogan, G.; Grotjahn, D. B. General Catalyst Control of the Monoisomerization of 1-Alkenes to Trans -2Alkenes. J. Am. Chem. Soc. 2014, 136 (4), 1226-1229. https://doi.org/10.1021/ja411438d.

(70) Mantilli, L.; Mazet, C. Iridium-Catalyzed Isomerization of Primary Allylic Alcohols under Mild Reaction Conditions. Tetrahedron Lett. 2009, 50 (28), 4141-4144.

https://doi.org/10.1016/j.tetlet.2009.04.130.

(71) Mantilli, L.; Gérard, D.; Torche, S.; Besnard, C.; Mazet, C. Iridium-Catalyzed Asymmetric Isomerization of Primary Allylic Alcohols. Angew. Chem. Int. Ed. 2009, 48 (28), 5143-5147. https://doi.org/10.1002/anie.200901863.

(72) Arai, N.; Sato, K.; Azuma, K.; Ohkuma, T. Enantioselective Isomerization of Primary Allylic Alcohols into Chiral Aldehydes with the Tol-Binap/Dbapen/Ruthenium(II) Catalyst. Angew. Chem. Int. Ed. 2013, 52 (29), 7500-7504. https://doi.org/10.1002/anie.201303423.

(73) Manzini, S.; Nelson, D. J.; Nolan, S. P. A Highly Active Cationic Ruthenium Complex for Alkene Isomerisation: A Catalyst for the Synthesis of High Value Molecules. ChemCatChem 2013, 5 (10), 2848-2851. https://doi.org/10.1002/cctc.201300396.

(74) Manzini, S.; Poater, A.; Nelson, D. J.; Cavallo, L.; Nolan, S. P. How Phenyl Makes a Difference: Mechanistic Insights into 
the Ruthenium( II )-Catalysed Isomerisation of Allylic Alcohols. Chem Sci 2014, 5 (1), 180-188.

https://doi.org/10.1039/C3SC52612G.

(75) Stewart, I. C.; Douglas, C. J.; Grubbs, R. H. Increased Efficiency in Cross-Metathesis Reactions of Sterically Hindered Olefins. Org. Lett. 2008, 10 (3), 441-444. https://doi.org/10.1021/ol702624n.

(76) Stewart, I. C.; Benitez, D.; O'Leary, D. J.; Tkatchouk, E.; Day, M. W.; Goddard, W. A.; Grubbs, R. H. Conformations of N-
Heterocyclic Carbene Ligands in Ruthenium Complexes Relevant to Olefin Metathesis. J. Am. Chem. Soc. 2009, 131 (5), 19311938. https://doi.org/10.1021/ja8078913.

(77) BouzBouz, S.; Simmons, R.; Cossy, J. Regioselective Cross-Metathesis Reaction Induced by Steric Hindrance. Org. Lett. 2004, 6 (20), 3465-3467. https://doi.org/10.1021/ol049079t.

\section{Table of Contents artwork}

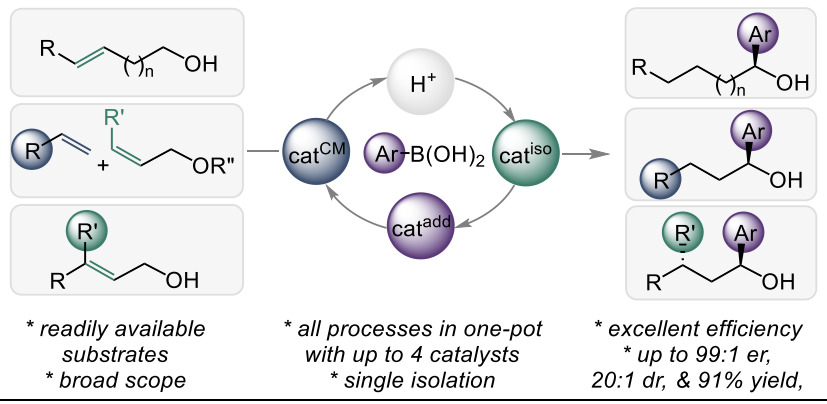

